\title{
Interface and Data Architecture for Query Preview in Networked Information Systems
}

\author{
Catherine Plaisant, Ben Shneiderman ${ }^{\#}$,Khoa Doan ${ }^{*}$ and Tom Bruns ${ }^{2}$ \\ Human-Computer Interaction Laboratory \\ University of Maryland Institute for Advanced Studies \\ \# Department of Computer Science and Institute for Systems Research \\ College Park, MD 20742 \\ http://www.cs.umd.edu/projects/hcil
}

Address correspondence to Catherine Plaisant,

HCIL/UMIACS A.V. Williams Building

University of Maryland, College Park, MD 20742

e-mail:plaisant @cs.umd.edu

phone: (301) 405 2725; fax: (301) 4056707

\section{to appear in ACM TOIS as a Practice and Experience Paper \\ Accepted version: September 1998}

First draft and technical report: April 1997

\begin{abstract}
There are numerous problems associated with formulating queries on networked information systems. These include increased data volume and complexity, accompanied by slow network access. This paper proposes a new approach to a network query user interfaces that consists of two phases: query preview and query refinement. This new approach is based on the concepts of dynamic queries and query previews, which guides users in rapidly and dynamically eliminating undesired records, reducing the data volume to a manageable size, and refining queries locally before submission over a network. Examples of two applications are given: a Restaurant Finder and a prototype for NASA's Earth Observing Systems--Data Information Systems (EOSDIS). Data architecture is discussed and user feedback is presented.
\end{abstract}

\footnotetext{
*Current Addresses: Khoa Doan, Best Software Inc., 11413 Isaac Newton Sq., Reston, VA 20190, Khoa_Doan@bestsoftware.com

2 Tom Bruns, Delorme Mapping, P.O.Box 298, Lower Main St., Freeport, ME 04032 e-mail: tbruns@ delorme.com
} 
Keywords: user interface, direct manipulation, dynamic query, information system, metadata, query preview, query refinement, science data, NASA EOSDIS.

\section{INTRODUCTION}

The exploration of networked information resources becomes increasingly difficult as the volume of data grows. We identified at least the following problems of information retrieval in networked environments:

- Data Volume: The amount of data available is rapidly increasing. For example, some sensor data in NASA's Earth Observing Systems is growing at the rate of gigabytes per day. Organizing and indexing the volume of new records is difficult. Since many users seek specific records, a rapid way to focus on information of interest is needed.

- Data Diversity: Data come in a variety of forms, such as text, image, audio, movies, or combinations of these. Some formats are application specific, making it difficult for search and retrieval tools to identify and categorize them.

- Slow Network Access: Slow network access is a well-known problem of information retrieval in networked environments. When network traffic is high, data transmission rates deteriorate. Therefore, user task completion is accelerated if the number of network accesses are reduced.

In this paper, we present a user interface to support efficient query formulation for networked information systems using dynamic queries and query previews.

Dynamic queries are an extension of graphical query interfaces based on aggregation/generalization hierarchies [1][2]. Dynamic query user interfaces apply the principles of direct manipulation and imply:

- Visual representation of the query

- Visual representation of the results

- Rapid, incremental, and reversible control of the query 
- $\quad$ Selection by pointing, not typing

- Immediate and continuous feedback

Dynamic queries involve the interactive control by users of visual query parameters that generate rapid, animated, and visual displays of database search results. As users adjust sliders or buttons, results are updated rapidly (within $100 \mathrm{msec}$ ).

The enthusiasm users have for query previews emanates from the sense of control they gain over the query. Empirical results have shown that dynamic queries are effective for novice and expert users to find trends and spot exceptions $[3,4,5]$.

Early implementations of dynamic queries used relatively small files of a few thousand records. They required the data to be stored in memory to guarantee rapid update of the display. We developed algorithms and data structures that allow larger files to be handled (up to 100,000 records) [6] but slow network performance and limited local memory are obstacles when trying to use dynamic queries for large distributed databases.

Query previews offer a solution to this problem. We describe a simple example of query previews, the Restaurant Finder, to illustrate the basic principles. Then the two-phase query formulation process and a system architecture are presented. A dynamic query user interface prototype for NASA's EOSDIS (Earth Observing Systems - Data Information Systems) is used to show how this approach has been applied. Evaluations from expert reviews and a controlled experiment are reported. Finally, related work and conclusions are presented.

\section{QUERY PREVIEWS}

Traditionally, there are two strategies for information seekers to obtain data from large information systems [7]. Analytical strategies depend on careful planning, recall of query terms, iterative query formulation, and examination of results. Browsing strategies depend on user recognition of relevant information, and therefore they are heuristic and opportunistic. Analytical strategies require users to have a good knowledge of the application domain, and be skillful in reasoning. Browsing strategies require less knowledge, but can be difficult when the volume of data is large. 
Keyword-oriented or form-based interfaces are widely used for formulating queries on networked information systems. They often generate zero-hit queries, or query results that contain a large number of results which users must browse. Users can limit how many results a query returns (e.g. 20) to limit the duration of the search but it is impossible to estimate how much data was not returned, and how representative of the entire search space the results are. Users also often fail to find data if appropriate keywords cannot be guessed.

Query previews combine browsing and querying. Summary data (such as the number of records for each attribute value) guide users to narrow the scope of their queries. The summary data, which varies with the database and application, provides an overview of the database from several perspectives. It is generally orders of magnitude smaller than the database itself, and can be downloaded quickly to drive a dynamic query interface locally on the user's machine. Therefore, query previews support a dynamic query user interface where the visual display of the summary is updated in real time in response to users' selections. Users can rapidly reduce the number of records to a manageable size.

Query previews empower users to perform more complex searches by using visual strategies and have many advantages:

- reduce zero-hit queries

- reduce network activity and browsing effort by preventing the retrieval of undesired records

- represent statistical information of the database visually to aid comprehension and exploration

- $\quad$ support dynamic queries, which aids users to discover database patterns and exceptions

- $\quad$ suitable to novice, intermittent, or frequent users

\section{A SIMPLE EXAMPLE OF QUERY PREVIEW: THE RESTAURANT FINDER}

The Restaurant Finder (Figure 1a and 1b) illustrates the concept of visual interaction with summary data, the essence of dynamic query previews. The Restaurant Finder is designed 
to help users identify restaurants that match certain criteria. Users first specify criteria of the restaurants they want, such as type of food or price range. This reduces the number of selected restaurants to a more manageable size (Figure $1 \mathrm{~b}$ ). The request is then submitted to the network, which retrieves more data on the selected restaurants. Users can then continue to refine their queries with additional, more specific, criteria.

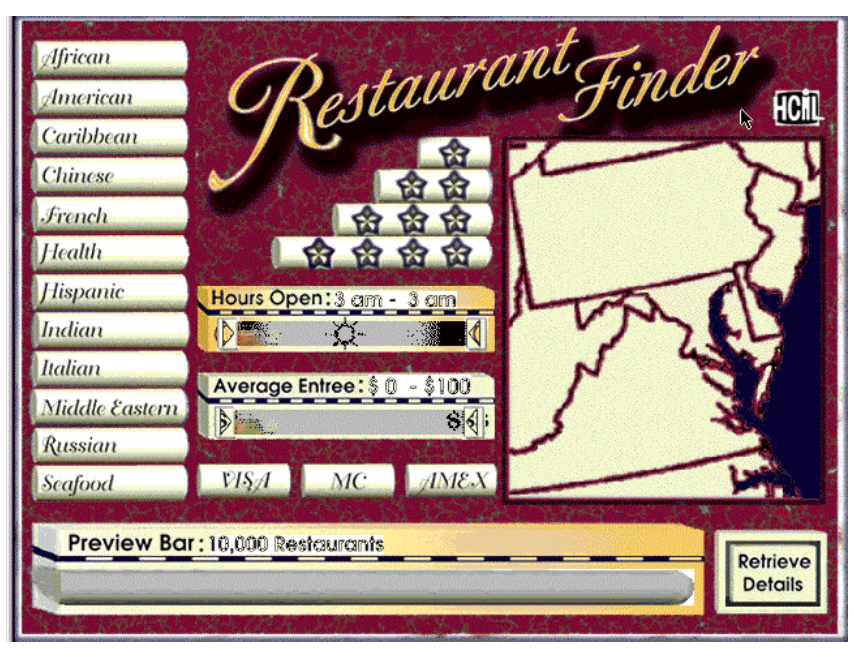

Figure 1a: Restaurant Finder. Users can choose an area on the map and make choices with buttons and sliders.

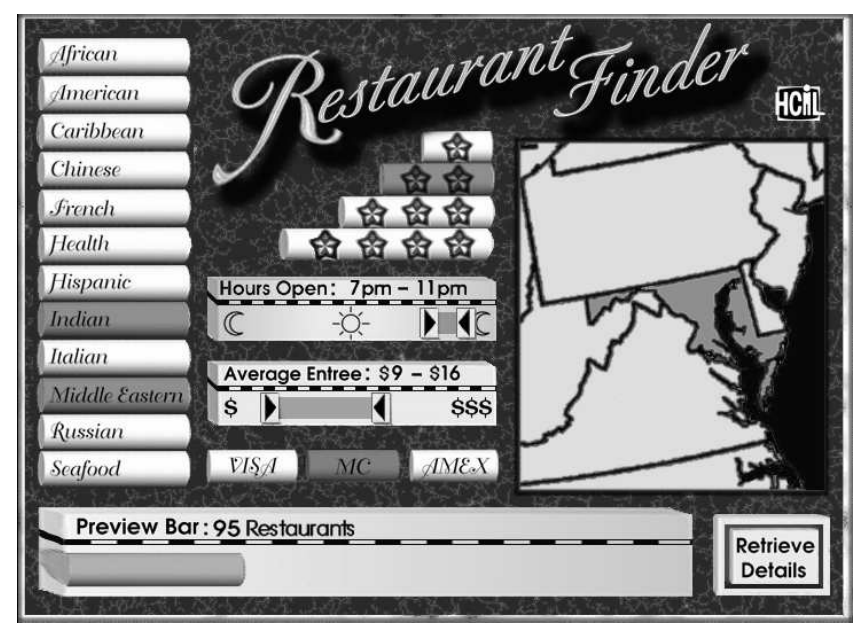

Figure 1b: Restaurant Finder. The user has now selected 2 cuisine types, a price range, and a geographical area, reducing the number of restaurant to review to 95 as shown on the result bar which is updated continuously as users adjust their queries. 
Consider a database of 50,000 restaurants in the mid-Atlantic region. The Restaurant Finder's user interface provides sliders and buttons for selecting desired cuisine, range of cost, range of hours, geographic regions, rating, and accepted charge cards. As selections are made, the result bar shown at the bottom of the screen changes length proportionally to the number of selected restaurants that satisfy the users' selection (possibly thousands of restaurants). Zero-hit queries are eliminated: users can quickly see if there are any Chinese restaurants open after midnight and they will rapidly realize that there are no cheap French restaurants in the DC area. Database distributions are visible: users may discover that there are more Chinese restaurants than Italian restaurants, but more Italian restaurants are open after midnight. In the query preview, only summary data is downloaded from the network, allowing real time interaction and eliminating network delays until a useful subset of the data has been identified. Then more details will be downloaded from the network about this subset (e.g. geographical location indicated on a zoom-able local map, data for parking availability, number of seats, or handicapped access) to allow users to refine their query. Finally users can click on individual restaurants and review menus and directions to make the final selection.

\section{MAIN EXAMPLE AND PROTOTYPE: THE CASE OF EOSDIS}

We use the NASA's Earth Observing System Data Information System (EOSDIS) to illustrate our two-phase query preview approach.

\section{EOSDIS SCIENCE DATA}

Diverse users (scientists, teachers, students etc.) can retrieve earth science data from hundreds of thousands of datasets. Datasets, named collections of data with authoritative metadata, contain pictures, measurements, or processed data, from nine data centers around the country. Standard EOSDIS metadata includes spatial coverage, time coverage, type of data, sensor type, campaign name, level of processing etc. Classic form fill-in interfaces for EOSDIS (Figure 2) permit searches of the already large holdings but zero-hit 
queries are a problem and it is difficult to estimate how much data is available on a given topic and what to do to increase or reduce the result set.

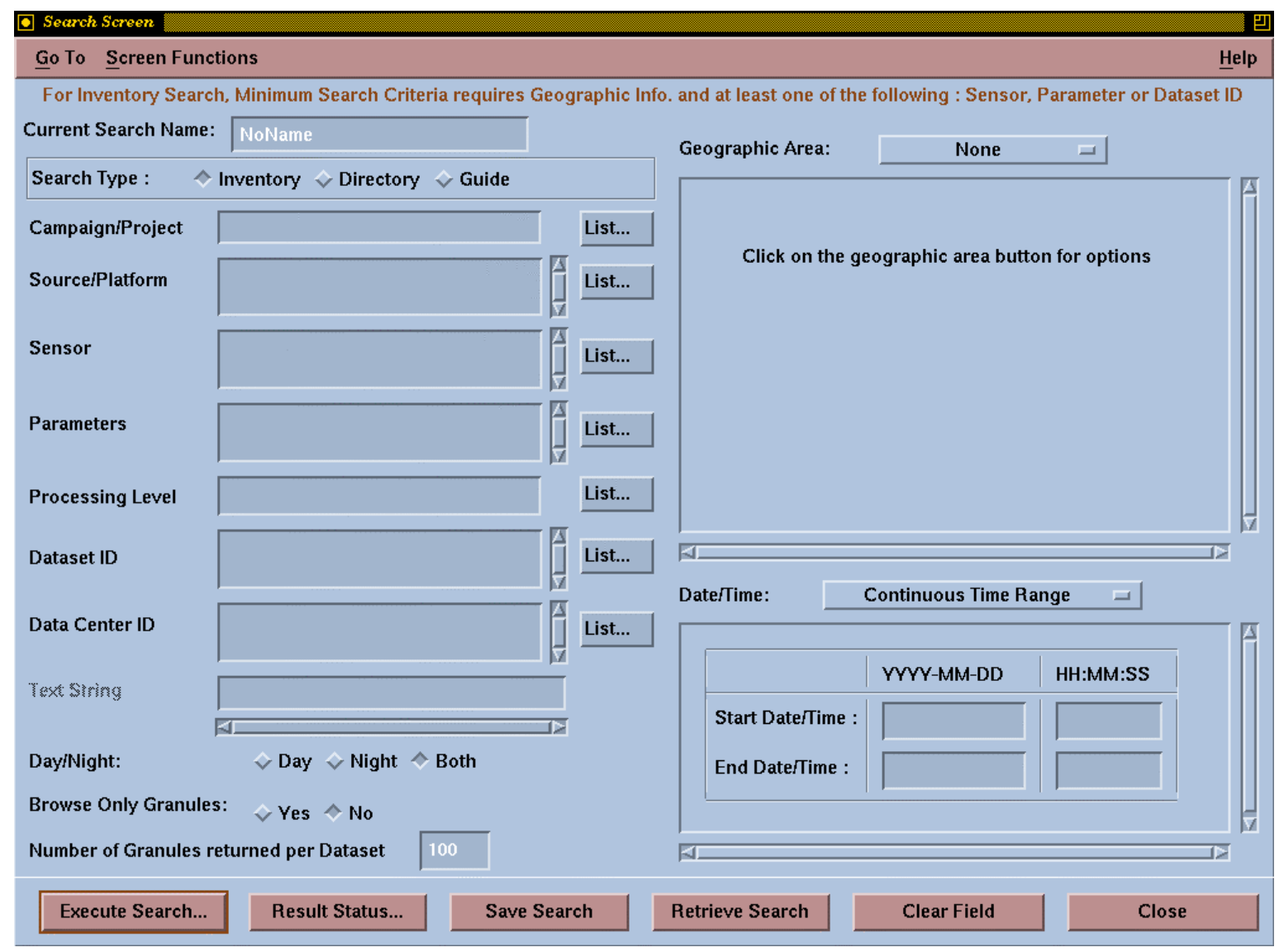

Figure 2: Classic form fill-in interfaces for EOSDIS (Figure 2) permit searches of the already large holdings but zero-hit queries are a problem and it is difficult to estimate how much data is available on a given topic.

\section{PROTOTYPES}

An early version of our two phase approach was implemented in Visual Basic [8]. Then a more complete prototype was implemented in Tcl/Tk (also available in video [9]) and more recently a working Java implementation was prepared on the World-Wide Web (WWW) [10]. The interface consists of two phases: query preview and query refinement. 


\section{EOSDIS QUERY PREVIEW}

In the query preview (Figure 3), users select rough ranges for three attributes: geographical location (a world map with 12 regions is shown at the top of the screen), parameters (a menu list of parameters such as vegetation, land classification or precipitation), and temporal coverage (in the lower right). The spatial coverage of datasets is generalized into continents and oceans. The temporal coverage is defined by discrete years.

The number of datasets for each parameter, region, and year is shown on preview bars. The length of the preview bars is proportional to the number of the datasets containing data corresponding to the attribute value. At a glance users can see that the datasets seem to cover all areas of the globe, but there is more data on North America than South America. Users can also see that parameters and years are covered relatively uniformly in this hypothetical EOSDIS dataset collection. The result preview bar, at the bottom of the interface, displays the total number of datasets.

Only rough queries are possible since the spatial coverage of datasets are generalized into continents and oceans and the temporal coverage is defined by discrete years.

A query is formulated by selecting attribute values. As each value is selected, the preview bars in the other attribute groups adjust to reflect the number of datasets available.

For example, users might be interested only in datasets that contain data for North America, which are selected by clicking on the North America checkbox (left of the map) or by clicking on the image of North America on the map. All the preview bars changes in a fraction of a second (see Figure $3 b$ ) to reflect the distribution of datasets for North America only. The result preview bar at the bottom changes size to indicate the number of datasets for North America (660 in this example).

Users continue to define a query by selecting from other attribute value groups. In this example, users pick the two largest attribute values for North America, "Vegetation" and "Land Classification" (see Figure 3b and c). The preview bars in the spatial and year attribute value groups adjust to reflect the new query. 
The OR operation is used within attribute value groups, the AND operation between attribute value groups [1]. Those AND/OR operations are made visible by the behavior of the bars which become smaller when an attribute value is specified for the first time (e.g. picking the first year) while becoming longer when additional values are added for a given attribute (e.g. when more years are added). This conjunction of disjunctions design handles many queries conveniently and allows rapid exploration that reduces the need for some more complex boolean queries [1][11].

Users further reduce the number of selected datasets by choosing specific years, in the example 1986, 1987, and 1988, three years which have data as shown on the preview bar (Figure 3d). These selections change the number of datasets in the other attribute value groups, and the preview bars are updated.

When the "Submit" button is pressed the rough query is submitted to the EOSDIS search engine and the metadata of the datasets that satisfy the query is downloaded for the query refinement phase. In the example the query preview phase narrowed the search to 66 datasets. 

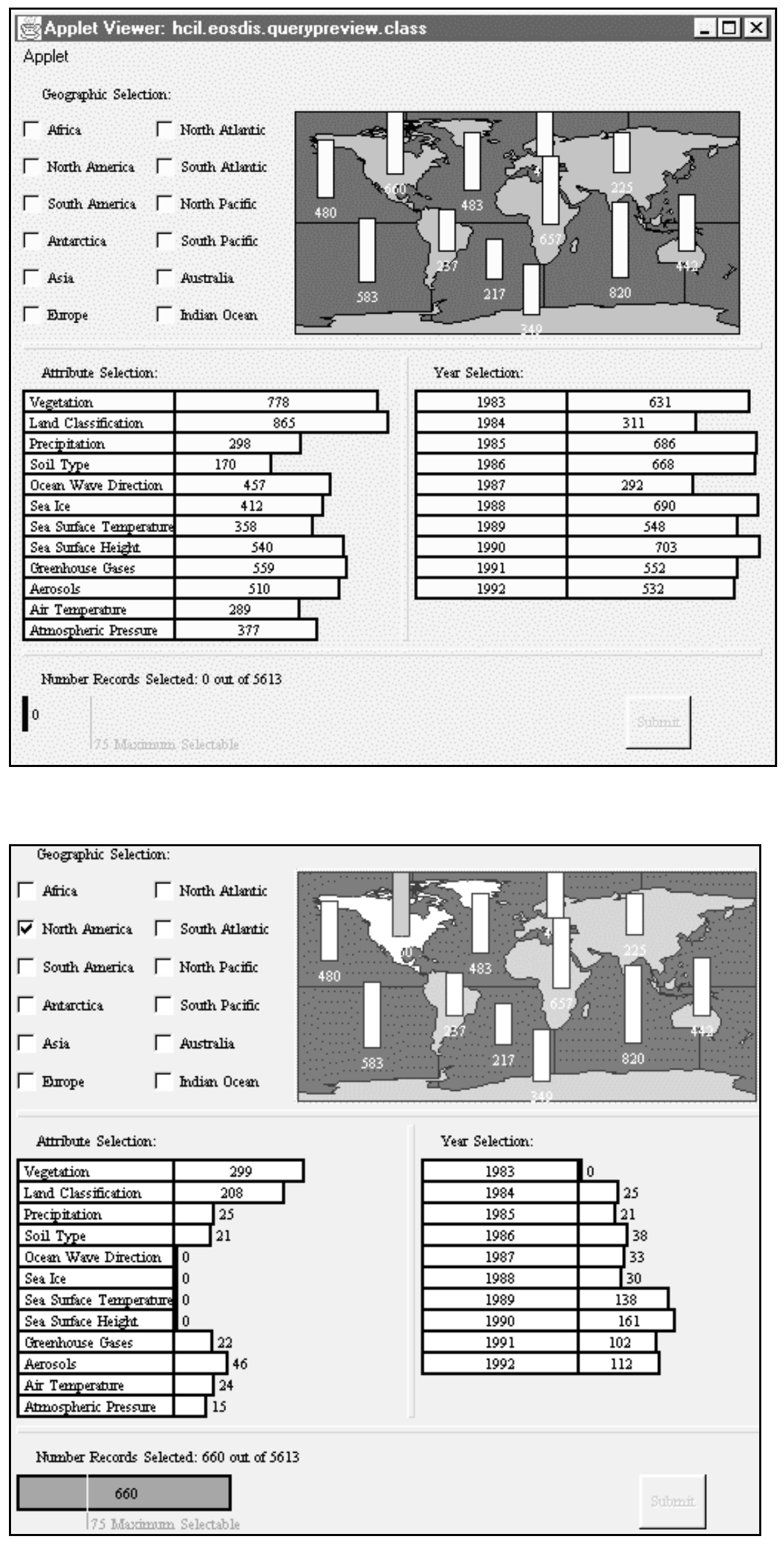

Figure 3a: The query preview screen displays summary data on preview bars. Users learn about the holdings of the collection and can make selections over a few parameters (here geographic, environmental parameter and year).

Figure 3b: The query preview screen displays summary data on preview bars. Users learn about the holdings of the collection and can make selections over a few parameters (geographic, environmental parameter and year). Here the user has selected North America and all preview bars are updated (using a logarithmic scale). 

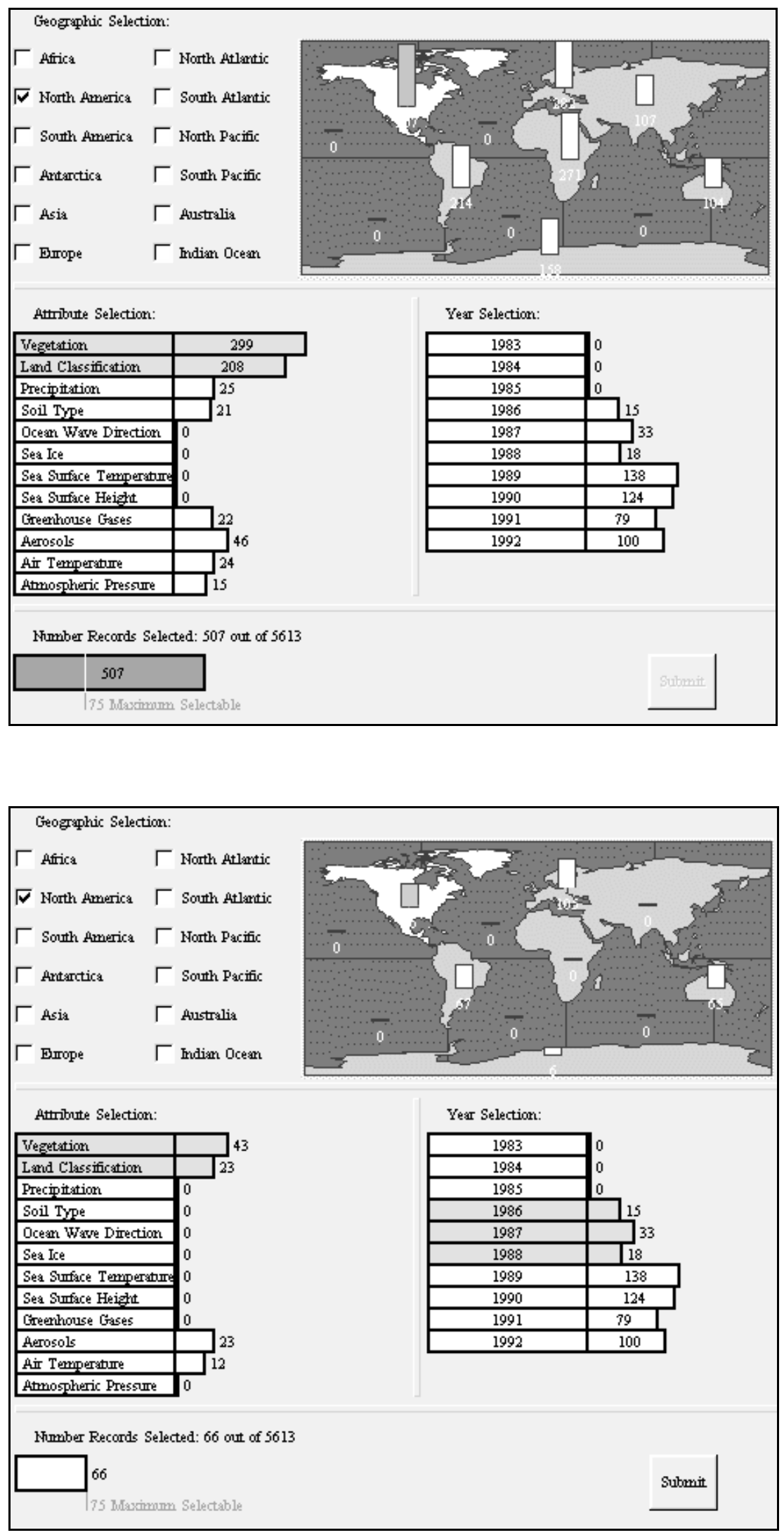

Figure 3c: Vegetation and Land Classification are now selected. The preview bars shows which years have data.
Figure 3d: Three years (1986, 1987, and 1988) have been selected. The result bar shows that an estimated 66 datasets will satisfy this query (the scale is logarithmic). The query can now be submitted. 


\section{EOSDIS QUERY REFINEMENT}

The query refinement interface supports dynamic queries over the metadata, i.e. over all the attributes of the datasets. These include: the detailed spatial extent and temporal interval, parameters measured in the dataset, the sensor used to generate the dataset, the platform on which the sensor resides, the project with which the platform is associated, the data archive center where the data is stored, and data processing level which indicates raw sensor data (level 0) to highly processed data (level 4).

A temporal overview of the datasets is given in the top left (Figure 4a). Each dataset is now individually represented by a selectable line. Controls are provided to select values for the common attributes: the data archive center, project, platform, sensor, and data processing level. Beside those common attributes additional attributes can be included in the metadata but since the number of attributes may be large, menu access needs to be provided for those less common attributes. At the bottom of the screen a table lists all the datasets and gives exact values for the attributes.

In the refinement phase of the query, users can select precise values for the attributes. The map, already zoomed to the area selected in the query preview, should be zoom-able to allow precise selection. The time line of the overview, already narrowed to the years selected in the query preview, can be re-scaled to specify narrower periods of interest. 


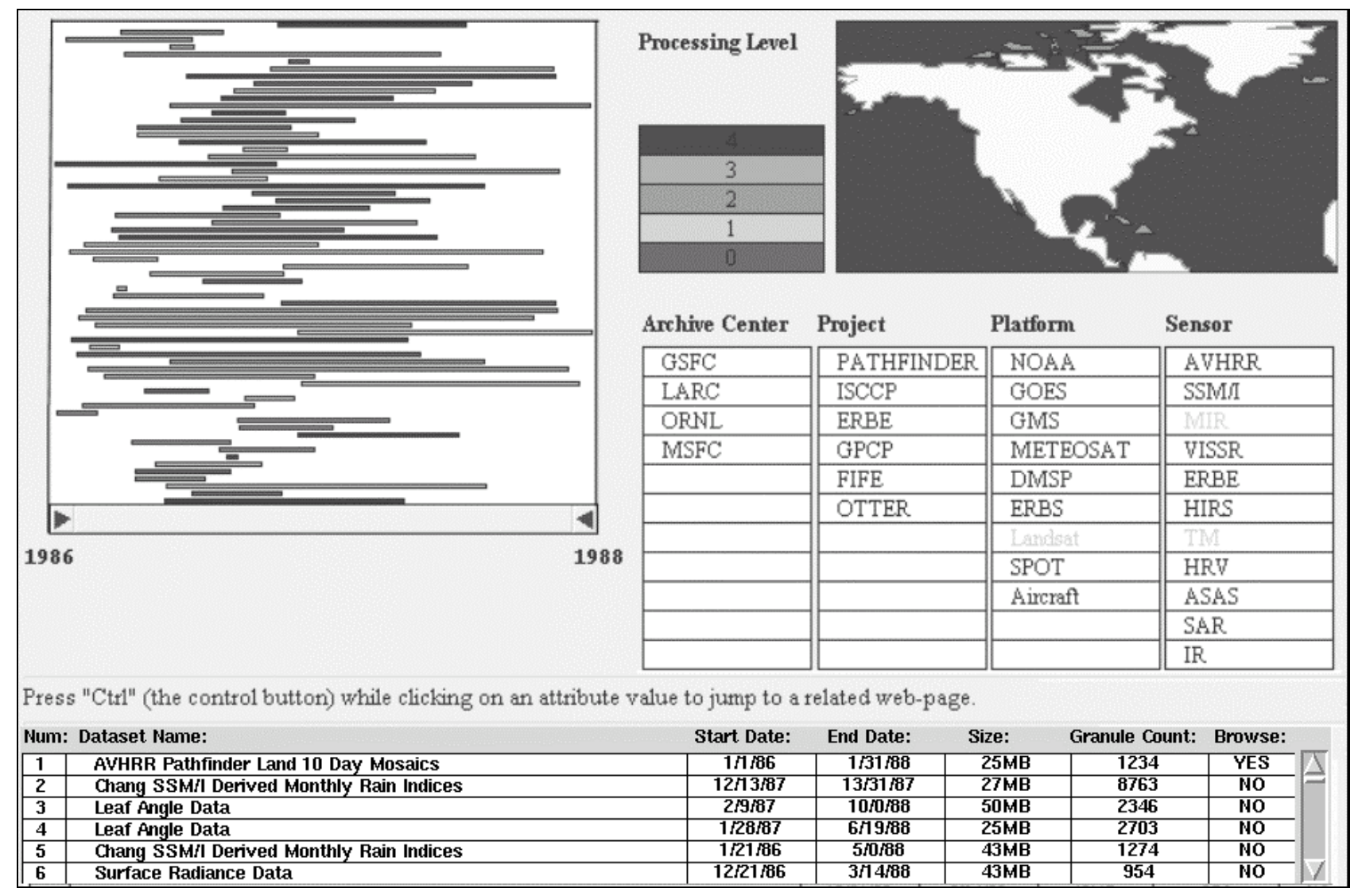

Figure 4a: In the query refinement users can browse all the information

\section{about individual datasets. The result set can be narrowed again by}

making more precise selections on more attributes.

In this second dynamic query interface the result of the query is immediately visualized on the overview. As attribute values are selected the number of lines on the overview changes to reflect the query in a few milliseconds since there is no access to the network.

All controls are tightly coupled to:

- Describe selected datasets. When users click on a dataset of the timeline overview, the corresponding attribute values are highlighted on all controls: e.g. the sensor is highlighted, the spatial coverage shown on the map, the row of the dataset table is highlighted and scrolled to the front if needed (Figure $4 \mathrm{~b}$ ) 
- Indicate valid values. Once some attribute values have been selected, controls can reflect the now invalid values by graying them out (e.g. selecting a platform will most likely eliminate some of the sensors which will become grayed out). This can be achieved by analyzing the metadata of the datasets.

In Figure $4 \mathrm{c}$ the number of datasets was reduced by selecting the processing levels 2 and 3, two archive centers, and three projects. More details about a dataset such as descriptive information and sample data can be retrieved on demand from the network before the decision to download a full dataset is made. The Java implementation also illustrates the benefit of the World-Wide Web by allowing interface objects to act as links to relevant WWW information sources. For example, each platform name is linked to a NASA page describing that platform.

\section{SYSTEM ARCHITECTURE}

The architecture supporting the two-phase query formulation consists of three layers: interface, local storage, and network (Figure 5).

At the interface layer, users formulate and refine queries as described above. The query preview and query refinement interfaces provide a visual representation of the preview statistics, selected datasets, and query parameters.

The local storage layer maintains the data used to drive the dynamic query interfaces of the interface layer. This data consists of a volume preview table (summary data that indicates the number of datasets for each attribute value and intersections) for the query preview, and dataset metadata for the query refinement. When users initiate a query preview session, the volume preview table is downloaded from the network databases.

The network layer is where the network activities take place. These network activities include updating the volume preview tables, providing the metadata for datasets selected from a query preview, retrieving the details of a dataset selected in the query refinement. 


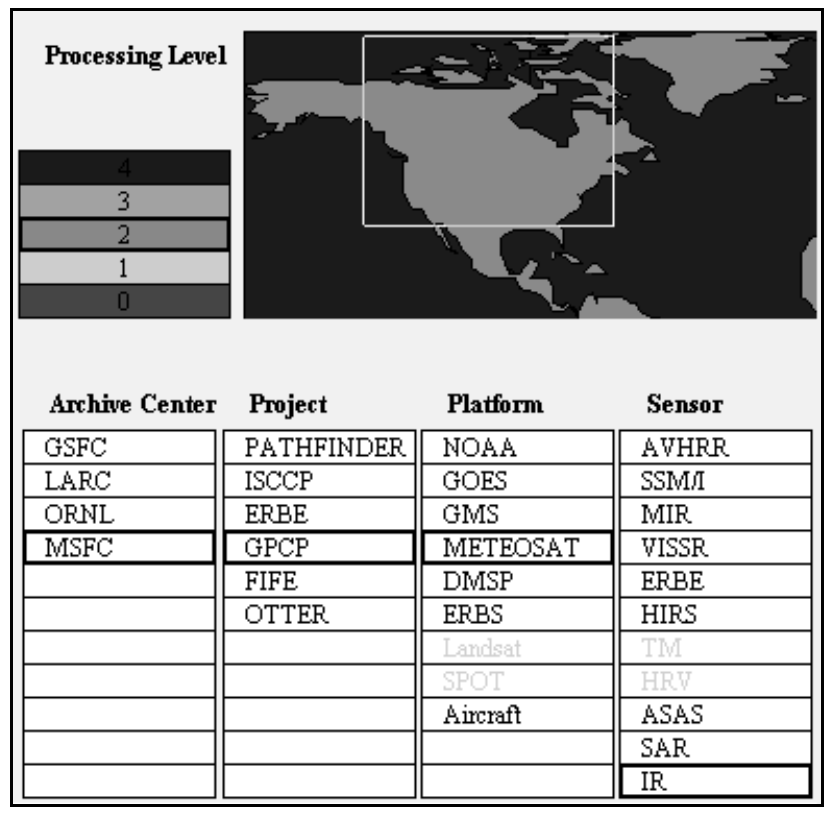

Figure 4b: Partial screen showing highlighted parameter values corresponding to a dataset selected on the timeline overview.

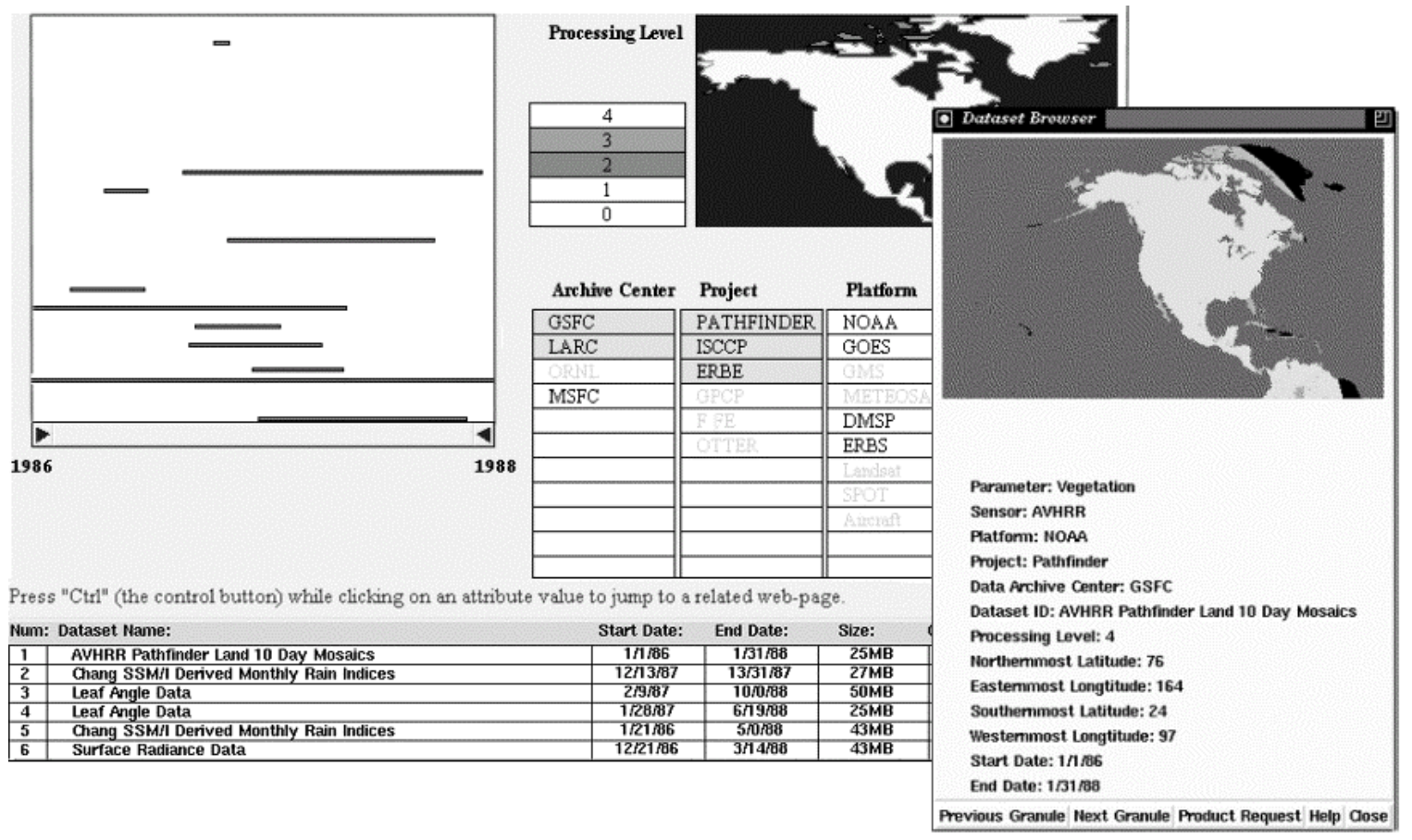

Figure 4c: Here the query has been refined by selecting 2 archive centers, 3 projects and 2 processing levels. More filtering could be done by zooming on the timeline or on the map. The timeline overview and the dataset table reflect the remaining datasets. Details and samples images can be downloaded from the network (window on the right) before the long process of ordering the large datasets. 


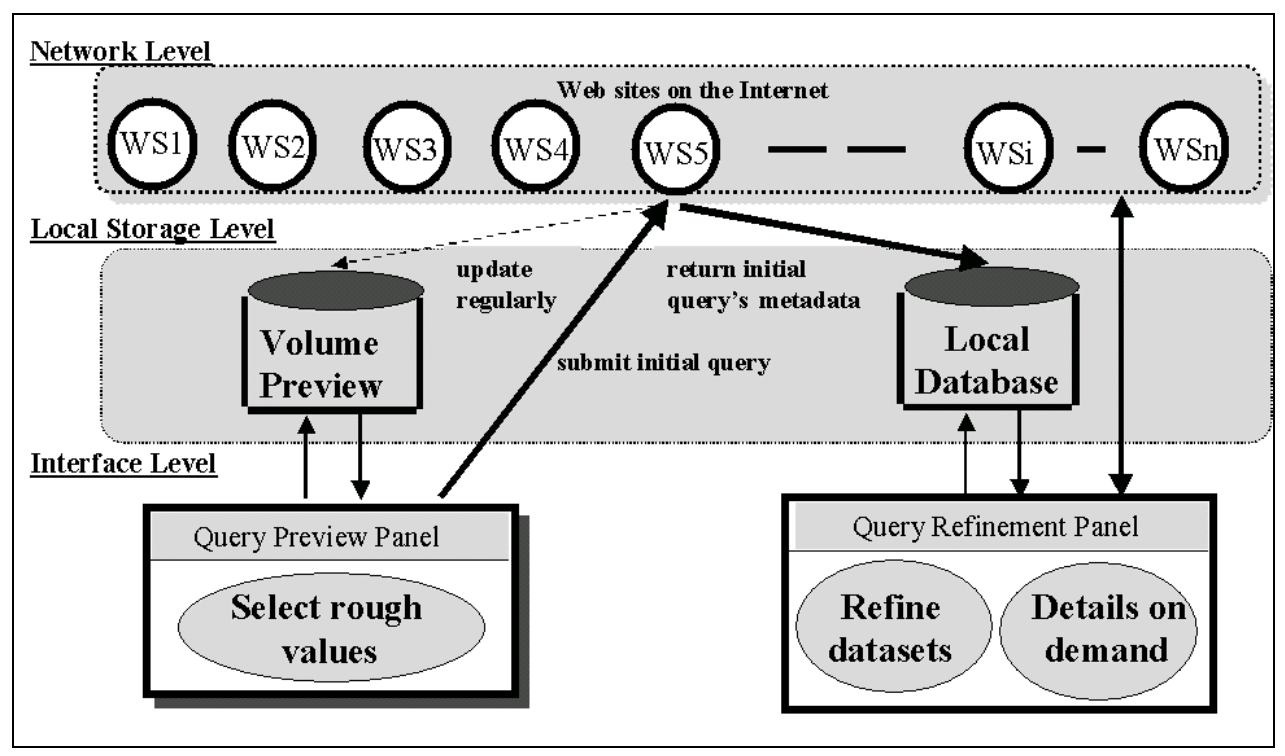

Figure 5: Architecture of two-phase dynamic query approach for networked information systems.

\section{Volume Preview table}

The size and dimensionality of the volume preview table is a function of the number of preview attributes and the number of discrete preview values for each attribute. Consider a Restaurant Finder with three preview attributes: cuisine type, rating, and accepted credit cards. Imagine five types of cuisine, four ratings, and two acceptable credit cards. In the simplifying case where each restaurant's attribute can only take a single value the volume preview table would be a five-by-four-by-two table, with a total of 40 combinations. But in our example of the Restaurant Finder, allowable credit cards may be grouped. The cells of the volume preview table must be independent so there must be cells for each possible combination of credit cards. Two credit cards create four possible combinations (including neither being acceptable), so the volume preview table has five-by-four-by-four or 80 combinations. Each cell in the table (i.e. each attribute value combination) holds an integer representing the number of restaurants in the database for that particular combination. In Figure 6 corresponding to the "three-star rated" restaurants, the cell for 3star Indian restaurant that accept Visa and MasterCard hold the value 98. Such tables are used to update widgets in the query preview interface. 


\begin{tabular}{|l|r|r|r|r|r|}
\cline { 2 - 6 } \multicolumn{1}{c|}{} & \multicolumn{1}{l|}{ French } & \multicolumn{1}{l|}{ Mexican } & \multicolumn{1}{l|}{ American } & \multicolumn{1}{l|}{ Indian } & \multicolumn{1}{l|}{ Italian } \\
\hline None & 18 & 9 & 5 & 6 & 8 \\
\hline Visa & 45 & 22 & 40 & 34 & 23 \\
\hline MC & 12 & 56 & 40 & 23 & 12 \\
\hline Visa \& MC & 80 & 90 & 120 & 98 & 160 \\
\hline
\end{tabular}

Figure 6: A slice of the volume preview table for an example Restaurant Finder. This 2D table results from specifying one of three preview attributes. In this case, the third attribute, rating, has been specified. This table is used to update preview bars in the query preview interface.

$\mathrm{N}$ preview attributes, yield an $\mathrm{N}$-dimensional volume preview table. The total size of the table is many orders of magnitude smaller than the size of the database, or the size of the datasets' metadata. Furthermore, the volume preview table does not change size as the database grows. Even if the database has billions of records, the size of the volume preview table allows it to be loaded into local high-speed storage to support dynamic queries in the query preview phase.

\section{Controlling the size of the table}

Nevertheless, the number of attributes and the number of the possible values needs to be carefully chosen if the objects being searched (e.g. restaurants or datasets) can take any combinations of values for their attributes. In the simple case of the Restaurant Finder, each restaurant could have a combination of credit cards. The interface widget only had 2 buttons for credit cards but the volume preview table needed 4 rows to represent the combinations. In the case of EOSDIS a given dataset can contain measurements of several parameters, covering several areas over several years. In the worst case (i.e. if all combinations are possible) the size of the preview table could become $2^{12} \times 2^{12} \times 2^{10}$ (for 12 areas, 12 parameters and 10 time 
periods) which would lead to megabytes of data, much too large to load over the network and use in the preview.

A first solution is to ignore in some way the possible combinations and count twice the datasets that have 2 parameters, once in each cell for each parameter it contains. This will result in correct individual preview bars (e.g. the preview bar for 1990 really gives the total number of datasets that have any data for that year) but inflate total result preview bar since some datasets are counted multiple times. This might be acceptable if combinations are a small proportion of the data, which is likely to be common because of the high granularity of the selections in the query preview.

A second more accurate solution to the problem is to analyze the number of combinations, either by looking at the type of attribute (e.g. year combinations are typically year ranges, reducing the number of combinations to 55 instead of 1024 for 10 values), or the distribution of the data itself (e.g. EOSDIS parameters are grouped into only a limited number of compatible combinations).

The first solution has the advantage of keeping the size of the volume preview very small (e.g. $12 \times 12 \times 10$ integers for our EOSDIS prototype, i.e. much smaller than the world map graphic), the second gives a more accurate preview but requires more time and space.

In our prototype we chose to simply duplicate datasets because we did not have access to large amounts of real EOSDIS metadata. The attributes were arbitrarily selected. However, it is not difficult to replace the set of attributes used in the prototype.

To summarize, volume preview tables can become large if combinations are to be previewed accurately or if large numbers of previewing attributes or attribute values are chosen. But the query preview technique can always be tailored by reducing the number of attributes or attribute values in the query preview. The size of the preview table can also be adapted to users' work environment (network speed, workstation type) or preferences. 


\section{Updating the volume preview table}

Since the data of the networked information system changes regularly, volume preview tables have to be updated. Our approach depends on the data providers being willing and able to produce and publish volume preview tables on a regular basis (weekly, daily or hourly depending on the application), or on third party businesses running series of queries to build the tables. Since the preview is only meant to enter rough queries it may be acceptable to use slightly out of date volume preview tables. The query preview needs to make clear that the preview bar sizes are an approximation of the real volume and give the "age" of the information used. When the rough query is submitted, the (up-to-date) databases are queried and will return up-to-date data for the query refinement. At this point the number of datasets returned might be slightly different than predicted by the query preview. This might be a problem when the query preview predicts zero hits while a new dataset that would answer the query has just been added to EOSDIS. This risk has to be evaluated and adequate scheduling of the updates enforced. The Cubetree implementation of datacubes [12] seems a promising data structure as it has efficient query update.

\section{Limiting the download of metadata}

Most users and data center staff will want to limit preview requests to those whose result set is small. The submit button can be disabled when the result set size is above a recommended level (75 in our prototype).

\section{LIMITATIONS OF THE CURRENT EOSDIS PROTOTYPE}

The present implementation of the query refinement interface has several limitations. The implementation of the query refinement overview will not scale up well when more than 100 datasets are returned from the query preview. The timeline of intervals will get too tall and occupy too much screen space if intervals are not allowed to overlap. Better methods of handling large numbers of intervals are needed. Possible directions include: zooming, optimizing the line packing to make use of screen space, or using line thickness to indicate overlaps. The quantitative and qualitative overview of the large number of datasets is needed 
to monitor their filtering but the ability to select individual lines is important when numbers have decreased enough to require browsing of individual datasets.

In our EOSDIS prototype the zooming and panning of the overview has no filtering effect but we have implemented other examples which demonstrate the benefit of the technique (e.g. for the Library of Congress historical special collections browsing [13]). Similarly the filtering by geographical location has not been developed yet in the query refinement. Zooming and selecting rectangular areas is easy but more sophisticated selection mechanisms used in geographical information systems are probably necessary.

The query preview allows users to specify the most common boolean queries (OR within attributes and AND between attributes). This is appropriate since the query preview is only meant to be a rough query, but more precise control over the boolean combinations need to be provided in the query refinement. Our current prototype does not offer such capability. Menu options can be provided to change the "behavior" of widgets, or graphical tools can be provided to allow boolean combination of the widgets [11].

\section{EVALUATION AND USER FEEDBACK}

\section{Expert user review}

The prototype dynamic query preview interface was presented to subjects as part of a Prototyping Workshop organized by Hughes Applied Information Systems (HAIS) in Landover, MD [14]. A dozen NASA earth scientists who use EOSDIS to extract data for their research participated in the evaluation and reviewed several querying interfaces during the day.

The hands-on review of our prototype lasted about an hour and a half. Groups were formed with two or three evaluators and an observer / note-taker in each group. They received no training but were given 5 directions or starting points to explore the prototype. For example, one direction was to "Examine the relationship between the map at the top and the data shown on the bottom half of the window. Try selecting a geographic region and various attributes. How are the data displayed." Evaluators were encouraged to "think aloud" during the session 
and their comments and suggestions were recorded. The 12 professionals reacted positively to the new concepts in the query preview and query refinement interfaces. They agreed that the visual feedback provided in the query preview interface allows users to understand the distribution of datasets. A group of evaluators recommended that it would be an effective tool for subjects who did not know what data was available. Others remarked that some users would not even need to go to the refinement phase as they would realize immediately that no data was available for them. The query preview interface was said to "allow users to select data, see relationships among data, and explore available resources".

Subjects said that they appreciated the time interval overview concept, liked to be able to select or deselect and see the changes in the overview. Subjects felt that the prototype "led the user", and was "an intuitive way to search data." Some users suggested that the map regions and selectable attributes be customizable so users could interact with information in which they are interested (different specialties may require different query preview attributes). At the time of the test the prototype was set to perform an AND operation within an attribute. This meant that clicking on 1991 just after a click on 1990 would result in all the bars being shorter (since it had restricted to the datasets which had data about 1990 AND 1991). After some confusion, all groups of evaluators were able to figure out that an AND was being performed by seeing the bars grow or shrink. But it was clear that they had expected the interface to perform an OR within attribute (i.e. retrieving all datasets having data from 1990 or 1991). This was an important change made to the prototype following the evaluation. This anecdote confirms that the visual feedback helped users understand the operations performed by the sytem.

After the evaluation, subjects were given a questionnaire and rated the interface positively. For a complete list of subject comments and questionnaire results, see [14].

\section{Controlled Experiment}

Twelve computer science students searched a database of films with a form fill-in interface. They were given only ten minutes of training in the use of the interfaces. The experimental treatments in this counterbalanced within-subjects design were presence or absence of a query 
preview [5]. The tasks simulated a complex browsing situation such as: "Find a PG-13 musical which was produced between 1991 and 1995, if no such film is available, find a war film from the same years with the same rating, if not, try a musical or a war film from 197091, and as the last possibility, try a comedy from 1970-95."

The query preview treatment showed whether or not there were any films satisfying the requirements, allowing subjects to rapidly explore alternatives. In the experiment, there were no lengthy network delays, so the time differences would be much larger if there were delays.

Subjects using the query preview took an average of only 36.2 seconds while others took 57.5 seconds $(\mathrm{p}<0.05)$ for tasks in which the query preview attributes were partially relevant. Stronger results, 24.4 seconds vs. 51.2 seconds $(\mathrm{p}<0.05)$, were obtained when the tasks closely matched the query preview attributes. This dramatic doubling of speed for query previews is a strong indication of its benefits, which will be even greater in the case of network delays. For tasks in which there was no match with the query preview attributes, there was only a $10 \%$ slowdown in performance.

Subjective satisfaction was statistically significantly higher for the query preview users, who rated the query preview interfaces higher on five questions: helpful? faster? enlightening? enjoyable? use it again? Subjects also made useful suggested improvements such as rapid ways to reset the query preview.

\section{SUMMARY AND DISCUSSION}

The two examples we described illustrate a query formulation process for networked information system consisting of two phases: query preview and query refinement.

\section{Query Preview}

In the query preview phase, users form a rough query by selecting rough values over a small number of attributes. The scope of the query is large, but the resolution is limited (see Figure 7). Summary data is maintained for each of the query preview attributes and intersections. 
The total number of items selected by the user's query is visualized on a result preview bar (at the bottom of the screen for both the EOSDIS and restaurant finder examples). Preview sizes can also be rendered on maps or charts, as illustrated in the EOSDIS prototype. These renderings must change within a fraction of a second in response to user input.

Selecting appropriate attribute values or categories rapidly reduces the data volume to a manageable size. Zero-hit queries are eliminated since users can spot them without issuing a query. Once users are satisfied with the formulated query, it is submitted over the network to the database. More details about individual records are then retrieved to refine the query.

\begin{tabular}{|l|l|l|}
\cline { 2 - 3 } \multicolumn{1}{c|}{} & Query preview & Query refinement \\
\hline Number of records & Very large & $\begin{array}{l}\text { Manageable (each one is } \\
\text { selectable for details-on- } \\
\text { demand) }\end{array}$ \\
\hline Number of attributes for & Few & $\begin{array}{l}\text { More or all of the } \\
\text { attributes }\end{array}$ \\
\hline Selection & & More precise or exact \\
values & Rough ranges or & values \\
\hline
\end{tabular}

Figure 7: A comparison table of the two phases of the query formulation process.

\section{Query refinement}

In the query refinement phase, users construct detailed queries over all database attributes, which are applied only to those records selected in the query preview. The scope of the query is smaller, but the resolution is finer. The interface provides access to all database attributes and their full range of values.

A characteristic of the query refinement phase is the rendering of each record in a graphical overview. The overview is closely related to the widgets used to refine a query, and reflects the query. By selecting appropriate values of relevant attributes, users continue to reduce the data volume and explore the correlation among the 
attributes through the visual feedback. Complete details can then be obtained at any time by accessing the database across the network for individual records.

\section{RELATED WORK}

An early proposal for volume previews in a database search is described in [15]. The "Dining out in Carlton" example was provided to illustrate a search technique (for a specific restaurant) based on the volume preview of the number of the available restaurants. However, query previews were not exploited to support dynamic queries and querying in networked information systems.

Retrieval by reformulation is a method that supports incremental query formation by building on query results [16]. Each time a user specifies a query, the system responds with query reformulation cues that give users an indication of how the repository is structured and what terms are used to index objects. Users can then incrementally improve a query by critiquing the results of previous queries. Rabbit [16] and Helgon [17] are examples of retrieval systems based on the retrieval by reformulation paradigm, which is also the basis of the two-phase query formulation approach.

Harvest [18] was designed and implemented to solve problems common to Internet users. It provides an integrated set of customizable tools for gathering information from diverse repositories, building topic-specific indexes, and searching. Harvest could be used to maintain and update the metadata servers where users can extract information and store it locally in order support dynamic queries in both the query preview and query refinement phases.

However, Harvest, just like other WWW browsers, still applies the traditional querying technique based on keywords. In order to express a complex query, a more visual query interface may be effective. Marmotta is a form-based tool used within WWW-clients to query networked databases [19]. The ease of use of form-based interfaces is preserved (users need not know the structure of the database). Within Marmotta, icons are used to present the domain of interest and the retrieval requests in a structured form-based interface. Icons are used in Marmotta to formulate a query. The system then translates 

problems or holes in the metadata that are not noticeable with classic form fill-in interfaces. This could be seen as a problem but we think that it will have a long term beneficial effect on the quality of the metadata as data providers will be compelled to produce more complete metadata. Our experience with the Global Master Change Directory demonstrates that the concepts are feasible in a large operational system, such as the EOSDIS directory environment.

\section{ACKNOWLEDGMENTS}

This work is supported in part by NASA (NAG 52895 and NAGW 2777) and by the NSF grants NSF EEC 94-02384 and NSF IRI 96-15534. We thank Teresa Cronnell for her graphic design of the Restaurant Finder prototype.

\section{REFERENCES}

1. Weiland, W. and Shneiderman, B., A graphical query interface based on aggregation/generalization hierarchies, Information Systems, vol. 18, \#4, 1993, pages $215-232$.

2. Shneiderman, B., Dynamic queries for visual information seeking, IEEE Software 11, 6, 1994, pages 70-77.

3. Ahlberg, C. and Shneiderman, B., Visual information seeking: Tight coupling of dynamic query filters with starfield displays, Proc. of the ACM CHI94 Conf., 1994, pages 313-319.

4. Williamson, C. and Shneiderman, B., The dynamic HomeFinder: Evaluating dynamic queries in a real-estate information exploration system, Proc. ACM SIGIR '92 Conference, ACM, New York, NY, 1992, pages 338-346.

5. Tanin, E., Lotem, A., \& Haddadin, I., Shneiderman, B., Slaughter, L., Plaisant, C., Evaluation of query previews: User preference and performance, University of Maryland Computer Science technical report CS-TR-3879 (Dec. 97) 
6. Tanin, E., Beigel, R. and Shneiderman, B., Incremental data structures and algorithms for dynamic query interfaces, ACM SIGMOD Record 25, 4, Dec. 96, pages 21-24.

7. Marchionini. G., Information Seeking in Electronic Environments. Cambridge University Press, UK, 1995.

8. Doan, K., Plaisant, C. and Shneiderman, B., Query previews in networked information systems, Proc. of the Forum on Advances in Digital Libraries, IEEE Computer Society Press, 1996, pages 120-129.

9. Doan, K., Plaisant, C., Shneiderman, B. and Bruns, T., Query previews in networked information systems: a case study with NASA environment data, ACM SIGMOD Records, Vol. 26, No 1, March 1997, pages 75-81

10. Plaisant, C., Bruns, T., Doan, K.and Shneiderman, B., Query Previews in Networked Information Systems: the case of EOSDIS, In CHI 97 Technical Video Program, Atlanta, GA, 1997, ACM New York. (also in HCIL 1996 video reports, HCIL/UMIACS, University of Maryland).

11. Young, D. and Shneiderman, B., A graphical filter/flow representation of boolean queries: a prototype implementation and evaluation, Journal of American Society for Information Science, vol. 44, \#6, July 1993, pages 327-339.

12. Roussopolos, N., Kotidi, Y., Roussopolos, M., Cubetree: organization of and bulk incremental updates on data cube, Proc. ACM SIGMOD 97.

13. Plaisant, C, Marchionini, G., Komlodi, A., Bruns, T, Campbell, L., Bringing treasures to the surface: the case of the Library of Congress Digital Library Program, Proc. of CHI' 97, ACM New-York, March 1997, pages 518-525.

14. Poston, J., Prototype Workshop 2 (PW2) Results Report. Technical Report 167-TP-001001, ECS Development Team, Hughes Applied Information Systems, Landover MD, 1996.

15. Heppe, D. L., Edmondson, W. H. and Spence, R., Helping both the novice and advanced user in menu-driven information retrieval systems, Proc. of British HCI85 Conf., 1985., pages 92-101. 
16. Williams, M.D.,What makes RABBIT run?, International Journal of Man-Machine Studies 21, 1984, pages 333-335.

17. Fischer and Nieper-Lemke, H., HELGON: Extending the retrieval by reformulation paradigm, Proc. of ACM CHI'89 Conf. , 1989, pages 333-352.

18. Bowman, C. M., Danzig, P. B., Hardy, D. R., Manber, U. and Schwartz, M. F., The Harvest information discovery and access system, Proc. of the Second International Conf. on the World Wide Web, 1994, pages 763-771.

19. Capobianco, F., Mosconi, M. and Pagnin, L., Progressive HTTP-based querying of remote databases within the Marmotta iconic VQS, Proc. of the IEEE Workshop on Visualization, 199, pages122-125.

20. Veerasamy, A. and Navathe, S., Querying, navigating and visualizing a digital library catalog, Proc. of the Second International Conf. on the Theory and Practice of Digital Libraries, 1995 (URL: http://www.csdl.tamu.edu/DL95/)

21. Hearst, M., Tilebars: Visualization of term distribution information in full text information access, Proc. of ACM CHI 95 Conf. , Denver CO, 1995, pages 5966.

22. Mackinlay, J., Rao, R.and Card, S., An organic user interface for searching citation Links, Proc. of ACM CHI'95 Conf., 1995, pages 67-75.

23. Greene, S., Tanin, E., Plaisant, C., Shneiderman, B., Olsen, L., Major, G., Johns, S., The End of Zero-Hit Queries: Query Previews for NASA’s Global Change Master Directory, University of Maryland Computer Science Technical Report, CS-TR-3855 (Dec. 1997). 